Mol. Cryst. Liq. Cryst. 1984, Vol. 107, pp. 45-53

0026-8941/84/1072-0045/\$18.50/0

(C) 1984 Gordon and Breach, Science Publishers, Inc.

Printed in the United States of America

$\left(\right.$ BEDT-TTF) ${ }_{2}^{+} J_{3}^{-}$:A TWO-DIMENSIONAL ORGANIC METAL

K. BENDER ${ }^{+}$, K. DIETZ ${ }^{++}$, H. ENDRES ${ }^{++}$, H.W. HELBERG ${ }^{+++}$,

I. HENNIG ${ }^{+}$H.J. KELLER ${ }^{++}$, H.W. SCHA'FER ${ }^{++}$and D. SCHWEITZER ${ }^{+}$

+Max-Planck-Institut für Med. Forschung, Abteilung für Molekulare Physik, Jahnstr. 29, D-6900 Heidelberg, Germany

${ }^{++}$Anorganisch-Chemisches Institut der Universität Heidelberg, Im Neuenheimer Feld 270, D-6900 Heidelberg, Germany

${ }^{+++}$Drittes Physikalisches Institut der Universität Göttingen, Bürgerstr. 42-44, D-3400 Göttingen, Germany

Abstract Temperature-dependent thermopower-, dc- and microwave conductivity measurements on the two-dimensional organic metal (BEDT-TTF) ${ }_{2}^{+} \mathrm{J}_{3}^{-}$are reported.

\title{
INTRODUCTION
}

Recently Saito et al ${ }^{1,2}$ have demonstrated that the nonplanar structure of BEDT-TTF [bis(ethylendithiotetrathiofulvalene)] (see fig. 1) leads in electrochemically prepared radical salts of this donor to strong intermolecular contacts and exchanges in more than one direction. (BEDT-TTFF) ${ }_{2}^{+} \mathrm{ClO}_{4}^{-} \cdot(\text { TCE })_{0.5}$ crystal plates have the typical electric behaviour of a quasi two-dimensional organic metal ${ }^{1,2}$.<smiles>CC1(C)SC2=C(SC(=C3SC4=C(S3)SC(C)(C)C(C)(C)S4)S2)S1</smiles>

in a similar salt (BEDT-TTF) ${ }_{4}^{+} \cdot\left(\mathrm{ReO}_{4}\right)_{2}^{-3,4}$ the two-dimensionality is somewhat less marked but in this case Parkin et al ${ }^{3,4}$ reported a superconducting behaviour near $2 \mathrm{~K}$ at an isotropic pressure of about $4 \mathrm{Kbar}$. So, for the first time in an organic sulphur donor system superconductivity was observed. Nevertheless, the strong twodimensional couplings were reached in both systems by a parallel side-to-side arrangement of the donor molecules within the stacks ${ }^{5}$. 
In (BEDT-TTF $)_{2}^{+} \mathrm{J}_{3}^{-}$crystals, on the other hand, in addition to such a side-by-side arrangement of the BEDT-TTF molecules within the stacks, an additional coupling between different stacks occurs due to large dihedral angles between the planes of donor molecules in crystallographically different stacks ${ }^{6}$. This results in short $(\sim 3.5 A)$ interstack S-S contacts in addition to the somewhat large contacts within a stack. Therefore a nearly isotropic conductivity in the ab-plane of such crystals is observed with typical room temperature values between 60 and $250\left(s 2 \mathrm{~cm}^{-1}\right)$. In the direction perpendicular to this plane at least a 1000 times smaller value is observed. The temperature - dependent dc-conductivity and thermopower measurements ${ }^{6}$ showed that in the temperature range between 135 and $300 \mathrm{~K}$ the crystals behave like a two-dimensional metal, while at $135 \mathrm{~K}$ a phase transition occurs with a sharp decrease in the electrical conductivity.

Here we report in addition temperature-dependent microwave conductivity $(10 \mathrm{GHz})$ and thermopower measurements. These indicate that in the temperature range between 120 and $30 \mathrm{~K}$ small sample regions might exist in which a semi-metal behaviour occurs.

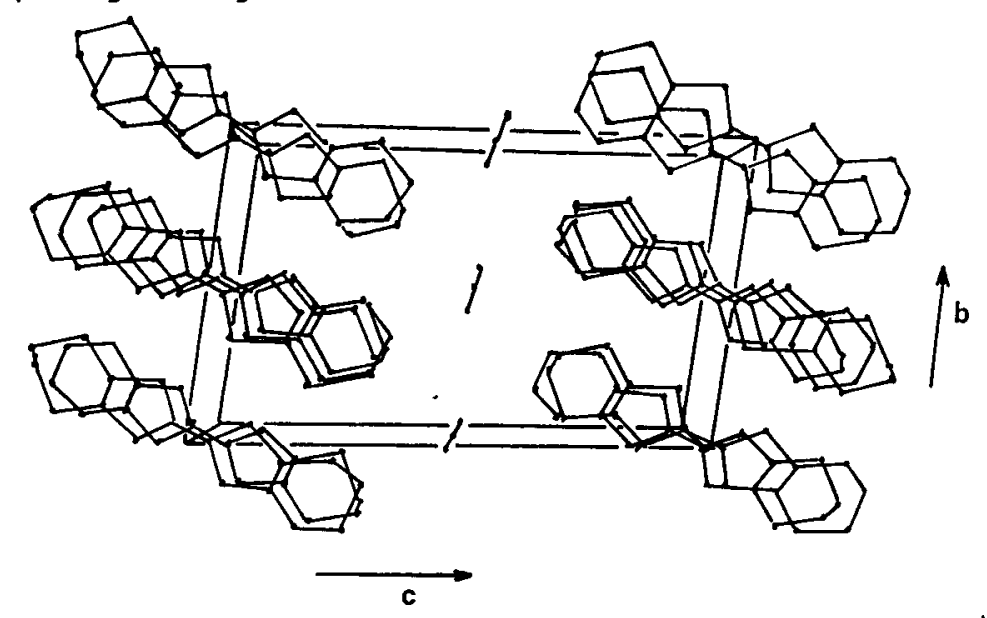

Fig. 2 Stereoscopic projection of the structure of $(B E D T-T T F)_{2}^{+} J_{3}^{-}$ along the stacking (a)-axis 


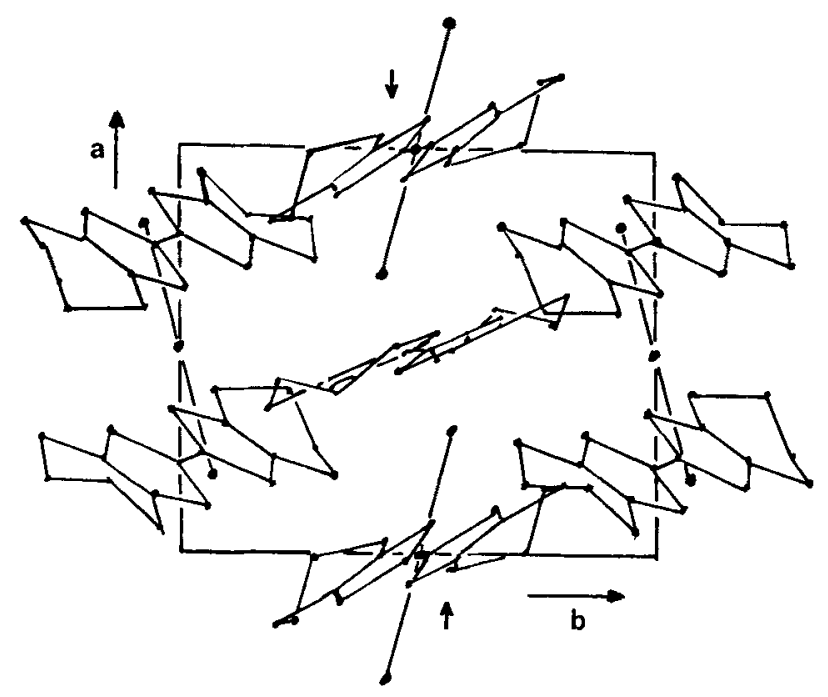

a)

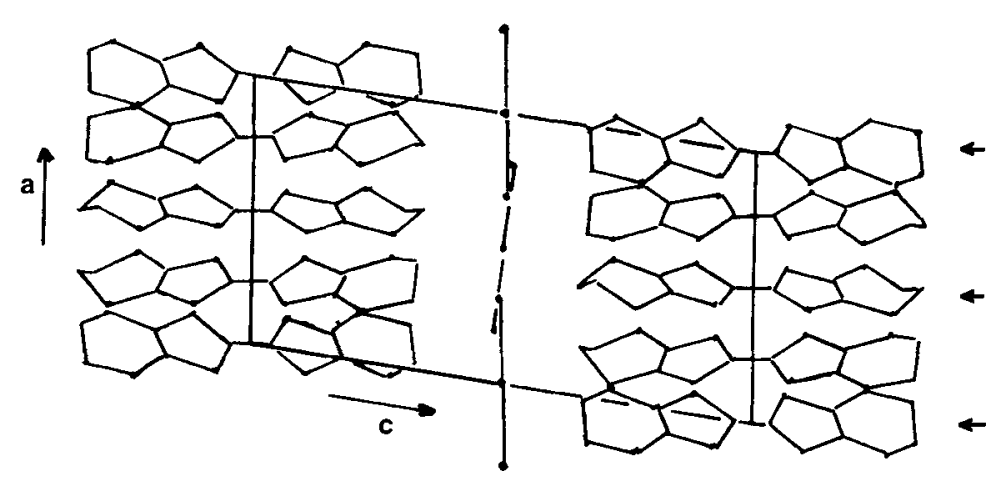

b)

Fiq. 3 Perpendicular projection of the unit cell of (BEDT-TTF) ${ }_{2}^{+} \mathrm{J}_{3}^{-}$ a): onto the ab plane b): onto the ac plane Arrows indicate the donor molecules of the same stack in both projections. 


\section{EXPERIMENTS AND RESULTS}

Fig. 2 shows the triclinic structure of (BEDT-TTF) ${ }_{2}^{+} \mathrm{J}_{3}^{-}$crystals seen along the $a$-axis (space group $P \bar{l}, a=9.211, b=10.850, c=17.488 \mathrm{~A}$; $\alpha=96.95, \beta=97.97$ and $\left.\gamma=90.75^{\circ}\right)$. The organic cations form two crystallographically different stacks parallel to the a-axis, as can be seen in the projection onto the ab-plane (fig. 3a). On the other hand the projection onto the ac-plane (fig. 3b) illustrates why the conductivity along the $\underline{c}$-axis is low.

The microwave conductivity measurements on (BEDT-TTF) ${ }_{2}^{+} \mathrm{J}_{3}^{-}$ crystals were carried out at $10 \mathrm{GHz}$ between 4 and $300 \mathrm{~K}$. The complex dielectric constant $\epsilon=\epsilon^{\prime}-\mathrm{i} \epsilon^{\prime \prime}$, where the imaginary part is connected with the microwave conductivity $\sigma=2 \pi \mathrm{f} \epsilon_{0} \epsilon^{\prime \prime}$ (assuming only dielectric losses, $\epsilon_{0}=8.854 \cdot 10^{-12}$ As/ $/ \mathrm{m}$ dielectric constant of vacuum, $f=$ frequency) was measured with the cavity perturbation method. Nearly quadratic single crystal plates with (001) faces were put into a cylindrical cavity, and the change in resonant frequency and $Q$-factor was measured. By exciting alternately the $T M_{010}$ and the $T E_{111}$ mode which have perpendicular E-field at the center of the cavity, it was possible to measure the conductivity along the a and $\underline{b}$ crystal axis in the same temperature run.

The $\underline{b}$ axis is almost perpendicular to the a axis $(\gamma=$ $\left.90.75^{\circ}\right)$. The axes may be distinguished by the absorption of light. In the visible range maximum absorption is found for light polarized parallel to the a axis (stack direction) ${ }^{7}$.

The real part $\epsilon^{\prime}$ can only be measured at low conductivities. At high conductivities the shift of the resonant frequency by the sample is governed only by the geometry of the sample. This allows directly the measurement of the depolarization factor of the actual sample orientation. Using this experimentally found depolarization factor (determined at $T \approx 140 \mathrm{~K}$ ) we improved the measurement accuracy at low conductivities $(T<120 \mathrm{~K}$ ). 


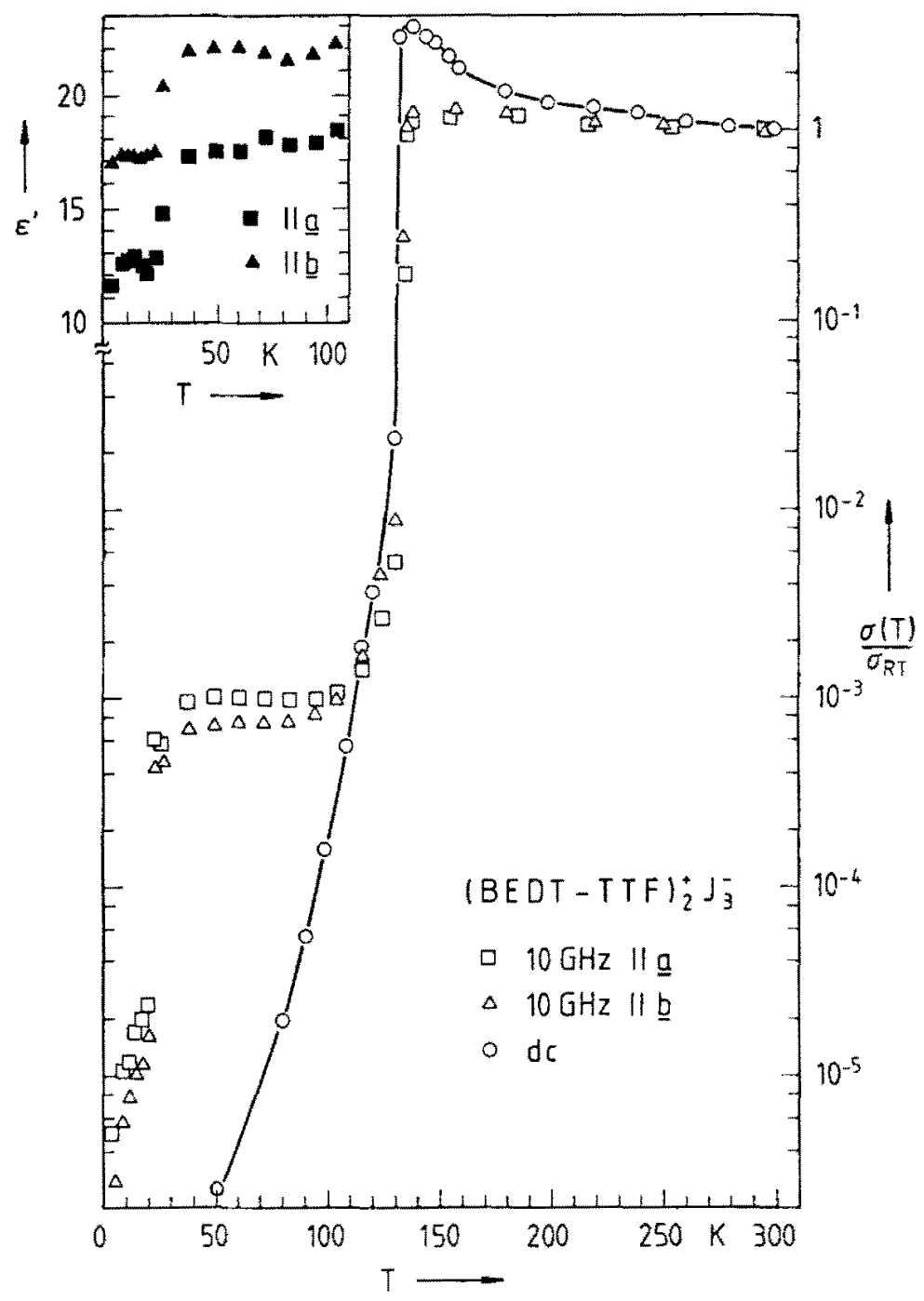

Fig. 4 Normalized temperature-dependent microwave conductivity $\sigma(\mathrm{T}) / \sigma_{\mathrm{RT}}$ of a (BEDT-TTF) ${ }^{+} \mathrm{J}_{3}^{-}$single crystal in a ( $\left.\mathrm{D}\right)$ and $\mathrm{b}(\Delta)$ direction. de-conductivity (o) from ref. 6 . Insert: dielectric constant ' $'$ of the same single crystal in a (I) and $b(\boldsymbol{A})$ direction as a function of temperature $T$. 
We found room temperature microwave conductivities of $\sigma_{R T, a}=30 . .60(\Omega \mathrm{cm})^{-1}$ and $\sigma_{R T, b}=50 . .90(\Omega \mathrm{cm})^{-1}$ in the a and $\underline{b}$ direction, respectively, and a conductivity anisotropy of $1 \leqslant \sigma_{R T, b} /$ ${ }^{\sigma} \mathrm{RT}, \mathrm{a} \leqslant 1.7$ : that means a higher conductivity in the b-direction perpendicular to the stack in good accordance to the dc-measurements where $1 \leqslant \sigma_{b}^{d c} / \sigma_{a}^{d c} \leqslant 2$ was found ${ }^{6}$. With decreasing temperature the conductivity increases slightly, until, at the transition around $135 \mathrm{~K}$, an abrupt drop is found (see fig. 4). By further lowering the temperature between $120 \mathrm{~K}$ and $40 \mathrm{~K}$, a plateau is reached with a microwave conductivity that is at least 1000 times smaller than in the metallic region above. Around $20 \mathrm{~K}$ another conductivity drop is observed, which suggests another phase transition. Below $20 \mathrm{~K}$ the conductivity decreases exponentially with an activation energy of about $0.8 \mathrm{meV}$.

At temperatures below $100 \mathrm{~K}$ it was also possible to determine the dielectric constant $\epsilon^{\prime}$ (see insert in fig. 4). In the plateau range between $100 \mathrm{~K}$ and $40 \mathrm{~K}$ constant values of ${ }^{\prime} \mathrm{a} \approx 18$ and ${ }^{\prime} \mathrm{b} \approx 22$ are observed for the $\underline{a}$ and $\underline{b}$ direction, respectively. By passing the second phase transition the dielectric constant decreases to ${ }^{\prime} a \neq 12$ and ${ }^{\prime} b 17$ below $20 \mathrm{~K}$.

The conductivity and dielectric constant in $\underline{a}$ and $\underline{b}$ direction behave in a remarkably similar way in the whole temperature range. Only around $135 K$ the conductivity drop is about 1.5 times larger in the $\underline{b}$ direction than in the a direction.

Fig. 5 shows the temperature dependence of the thermopower. In addition to earlier measurements ${ }^{6}$ the thermopower 5 is reported also below $100 \mathrm{~K}$. At temperatures above $100 \mathrm{~K}$ the earlier values of $\mathrm{S}^{6}$ are in very good agreement with those shown here. Below $100 \mathrm{~K}$ it can be seen that the thermopower $S$ changes from large negative to large positive values. This change occurs in the same temperature region where the microwave conductivity shows a constant value in contrast to the oc-conductivity. 


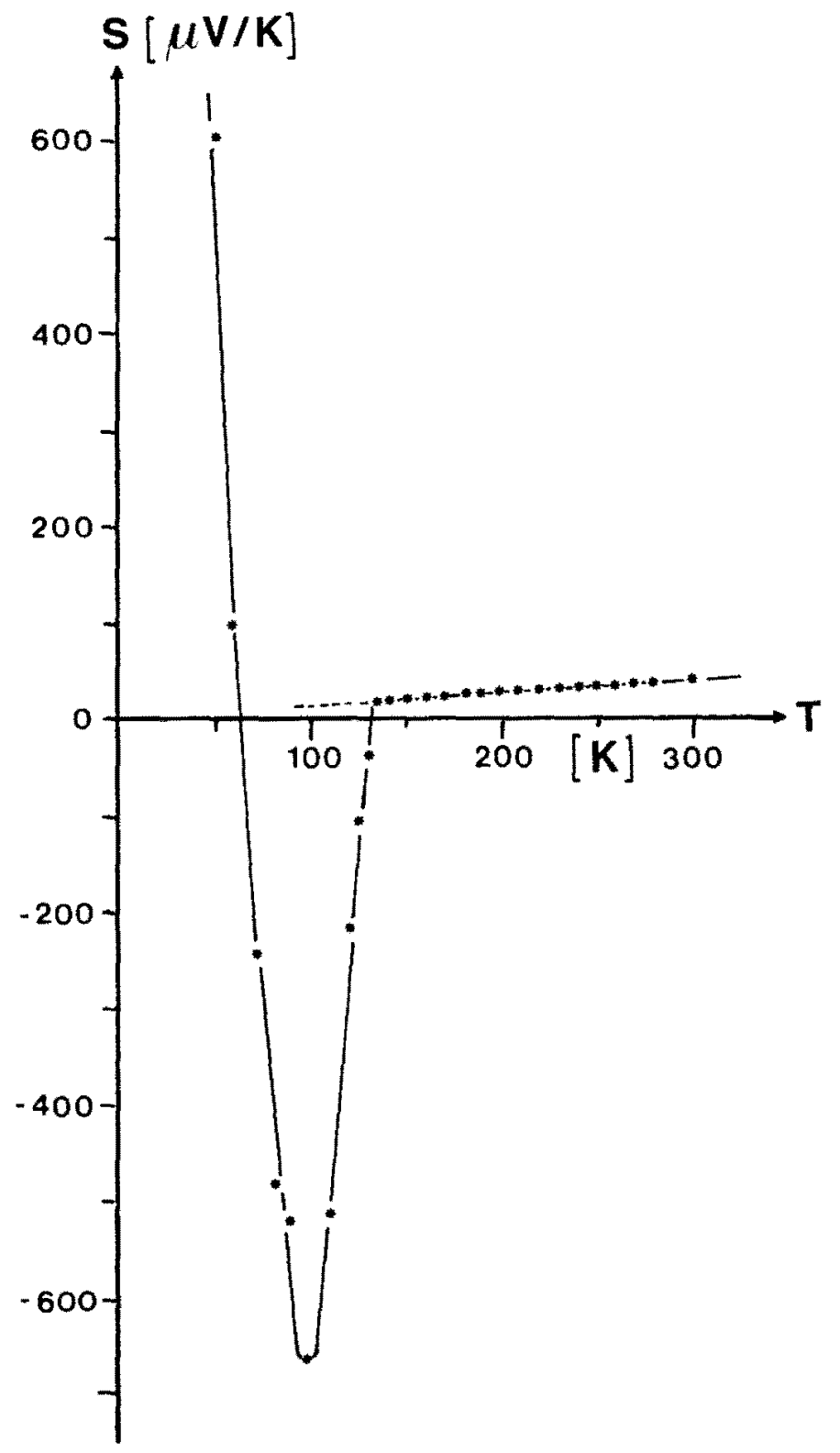
Fig. 5 Temperature- dependent thermopower $S$ of (BEDT-TTF ${ }_{2}^{+} J_{3}^{-}$
single crystals 


\section{DISCUSSION}

The room-temperature values of the dc- and microwave conductivities are similar. The importance of the two-dimensional network of $\mathrm{S}-\mathrm{S}$-contacts for the conductivity is emphasized by the fact that the conductivities in the b-direction is even somewhat higher than in the stack (a)-direction. This observation is in accordance with the structural finding that the interstack S-S contacts are shorter than the intrastack ones. A comparison of the temperature dependence of the microwave and dc-conductivity shows significant differences below $120 \mathrm{~K}$, while above $135 \mathrm{~K}$ both conductivities at dc- and $10 \mathrm{GHz}$ indicate a metallic region with a qualitatively similar temperature dependence. The linear temperature-dependent thermopower $\mathrm{S}$ above $135 \mathrm{~K}$ further confirms the metallic behaviour of the samples. The positive sign of $\mathrm{S}$ implies that the conductivity is dominated by holes. Below $120 \mathrm{~K}$ the dc-conductivity continues to decrease, while the microwave conductivity is constant down to about $30 \mathrm{~K}$. At $50 \mathrm{~K}$ the difference between both conductivities reaches about 3 orders of magnitude, and this ratio increases further to lower temperatures. The frequency dependence of the conductivity in the temperature region between 120 and $30 \mathrm{~K}$ might be due to the fact that, below the phase transition at $135 \mathrm{~K}$, still small sample regions exist in which a semi-metal behaviour occurs. This behaviour can only be observed in the microwave conductivity $(10 \mathrm{GHz})$ which yields the bulk conductivity. At dc, however, the observed conductivity is governed by dc-barriers between undisturbed areas. Another mechanism may be the excitation of pinned charge density waves which produce an increasing conductivity with higher frequencies. On the other hand, the thermopower $S$ indicates in the same temperature region that the dominant contribution to the conductivity changes from electrons to holes. This might be due to the fact that the number of carriers changes in the semiconducting regime, or 
that the mobility of the electrons and holes varies drastically, resulting in a much higher mobility of the holes.

Nevertheless, further investigations are necessary to clarify the microwave conductivity plateau and the change in the thermopower behaviour at around $100 \mathrm{~K}$.

\section{ACKNOWLEDGEMENT}

D.S. would like to thank Prof. M. Weger for very helpful discussions. This work has been supported by Stiftung Volkswagenwerk Hannover.

\section{REFERENCES}

1. G. Saito, T. Enoki, K. Toriumi and H. Inokuchi, Solid State Comm. 42, 557 (1982).

2. G. Saito, T. Enoki, H. Inokuchi and H. Kobayashi, Journal de Physique 44, Colloque C3, 1215, 1983.

3. S.S.P. Parkin, E.M. Engler, R.R. Schumaker, R. Lagier, V.Y. Lee, J.C. Scott, and R.L. Greene, Phys. Rev. Lett. 50, 270 (1983).

4. S.S.P. Parkin, E.M. Engler, R.R. Schumaker, R.Lagier, V.Y. Lee, J. Voiron, K. Carneiro, J.C. Scott and R.L. Greene, Journal de Physique 44, Colloque C3, 791, 1983.

5. M. Kobayashi, A. Kobayashi, Y. Sasaki, G. Saito, T. Enoki, H. Inokuchi, J. Am. Chem. Soc. 105, 297 (1983).

6. K. Bender, I. Hennig, D. Schweitzer, K. Dietz, H. Endres and H.J. Keller, Mol. Cryst. Liqu, in print.

7. Measurements of the tensor orientation of polarizability and absorption. H.W. Helberg, to be published. 